\title{
Numerical Modelling of the Sound Absorption Spectra for Bottleneck Dominated Porous Metallic Structures
}

\author{
${ }^{1}$ Otaru, A.J., ${ }^{1}$ Morvan, H.P. \& ${ }^{2}$ Kennedy, A.R. \\ ${ }^{1}$ Gas Turbine and Transmissions Research Centre, Faculty of Engineering, The University of Nottingham, \\ Nottingham NG7 2RD, UK. \\ ${ }^{2}$ Engineering Department, Lancaster University, Lancaster LA1 4YW, UK
}

\begin{abstract}
Numerical simulations are used to test the ability of several common equivalent fluid models to predict the sound absorption behaviour in porous metals with "bottleneck" type structures. Of these models, Wilson's relaxation model was found to be an excellent and overall best fit for multiple sources of experimental acoustic absorption data. Simulations, incorporating Wilson's model, were used to highlight the relative importance of key geometrical features of bottleneck structures on the normal incidence sound absorption spectrum. Simulations revealed significant improvements in absorption behaviour would be achieved, over a "benchmark" structure from the literature, by maximising the porosity $(0.8)$ and targeting a permeability in the range of $4.0 \times 10^{-10} \mathrm{~m}^{2}$. Such a modelling approach should provide a valuable tool in the optimisation of sound absorption performance and structural integrity, to meet applicationspecific requirements, for a genre of porous materials that offer a unique combination of acoustic absorption and load bearing capability.
\end{abstract}

Keywords: Porous Metal; Sound Absorption; Simulation.

\section{Introduction}

Glass wool, cotton, asbestos, fur felt, hemp flax and synthetic fibre (polyester, polypropylene, and Kevlar) materials are known to be very good absorbers of sound and inexpensive [1]. Their low heat resistance, high-moisture content and decrease in absorption potential over time [2], have limited their application for sound absorption across a wide range of engineering devices. Open-celled metallic structures with high specific surface area, low moisture content, high heat resistance and which are non-flammable, are commercially available [3-5] and are being considered, and in several instances used, in sound absorbing applications where the above attributes are important. Their unique combination of multi-functional performance (those of structural integrity and function) could thus see them replacing more complex constructions containing "traditional" sound absorbers. 
It is important, however, to compare the performance of porous metals with more "traditional" sound absorbers, such as glass wool and open cell polymer foams. Fig 1 does this, comparing the structural thickness dependent normal incidence absorption coefficient, also termed the ratio of sound intensity absorbed to incident sound intensity, at varying frequencies, for different porous absorbers from the literature. Images of the corresponding structures for the materials referred to in this figure are presented in Fig 2. Whilst the samples are not of identical thickness, the behaviour presented is representative of the relative performances of these materials. It is apparent, for this example, that "traditional" absorbers, such as glass wool fibres and melamine foams, show very high, and sustained, absorption coefficients, for frequencies above approximately $3000 \mathrm{~Hz}$.

Porous metals with interconnected porosity are often made by replicating these structures, for example by sintering metal fibres, or metallic coating of open cell polymer foams [6]. Technological limitations prevent attaining the same high levels of porosity and small cell sizes and offer limited potential to alter the pore morphology in-process. These structural differences tend to lead to lower flow resistivities for the metallic counterparts [7] and inferior absorbers. Fibre felts, or sintered metal fibres $[7,8]$ offer the closest matching performance to those of glass wool and polymer foams, as shown in Fig 1, but offer little or no structural function.

Fig 1 shows the absorption behaviour for a so-called "bottleneck-type" porous metal structure (from [9]) made by casting liquid metal into the spaces between a packed bed of dissolvable salt beads $[9,10]$. These structures are typified (shown in Fig 2) by porosities in the range of $55-80 \%$ and pores between 0.5 and $3 \mathrm{~mm}$ in diameter, connected by smaller "windows". Their much higher solid fraction than most other porous metal structures means that they offer credible structural function and energy absorbing capability, thus making them suitable candidates for multi-functional sound absorbers. The sound absorption for these materials [9] is good for frequencies within the quarter wavelength layer resonance absorption, but poor over the mid-frequency range $(2000-4500 \mathrm{~Hz})$. 


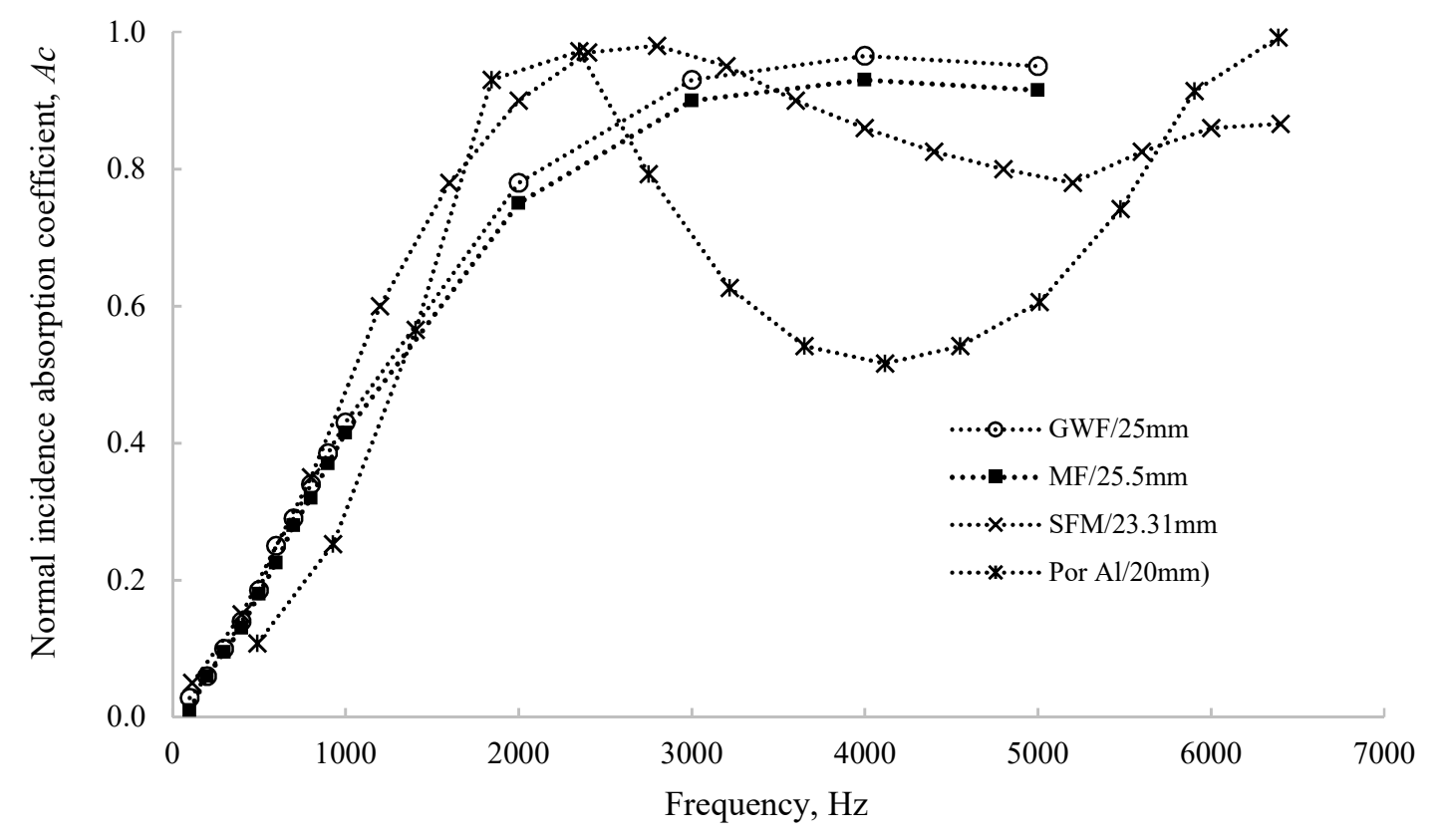

Fig 1. Plots of normal incidence absorption spectra against frequency for a number of different porous materials. Namely a $25.0 \mathrm{~mm}$ thick hard-backed glass wool fiber material (GWF, 98.7\% porosity [11]), $25.5 \mathrm{~mm}$ hardbacked melamine foam (MF, 99.3\% porosity [11]), $23.3 \mathrm{~mm}$ thick hard backed porous sintered fiber metal (SFM, $90.94 \%$ porosity [8]) and a $20 \mathrm{~mm}$ thick hard-backed porous aluminium with a bottleneck structures (Por $\mathrm{Al}, 60 \%$ porosity [9]).
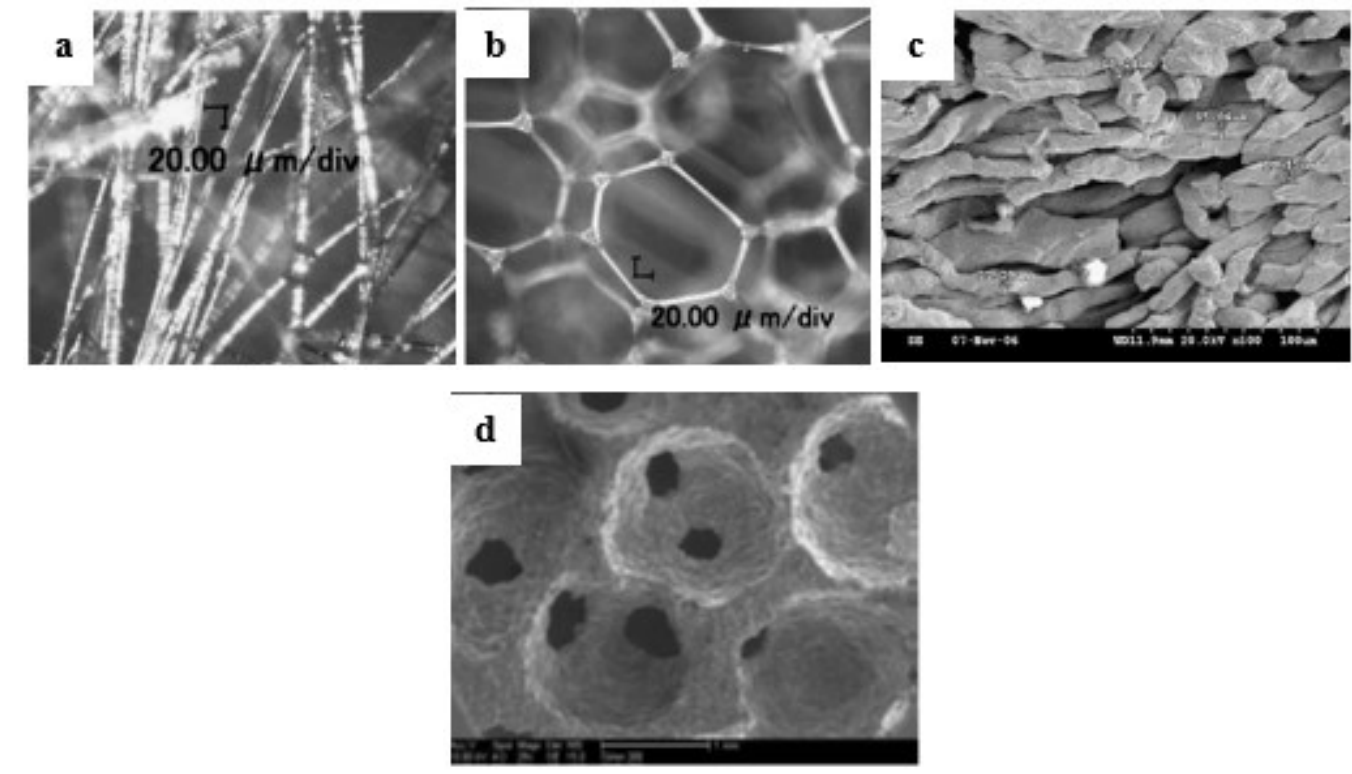

Fig 2. Optical microscopy images of (a) glass wool fiber material (GWF, [11]) (b) melamine foam (MF, [11]), (c) porous sintered fiber metal structure (SFM [8]) and (d) porous aluminium with bottleneck structure (Por Al, [9])

Previous research into sound absorption in porous metals with bottleneck-type structures has indicated that improvements in noise reduction coefficients can be achieved with modifications to the structure [12-14] including increasing porosity [13] and decreasing pore openings 
(window sizes) [14]. A more comprehensive understanding of the influence of key structural parameters specific to this porous metal type (for example, porosity, pore size and window size) is required, if the gap in performance to "traditional" sound absorbing materials is to be reduced. This study aims to begin this process by developing and demonstrating a reliable numerical simulation method for the prediction of the sound absorption performance of porous metals with bottleneck-type structures.

\section{Simulation methodology}

The simulation procedure for pressure wave propagation across porous structures was carried out with the following steps, namely: definition of pore structure-related and geometry parameters and variables, geometry creation, material selection, choice of physics and appropriate boundary conditions, meshing and solving within the pressure acoustic frequency domain module of COMSOL Multiphysics 5.2 $2^{\mathrm{TM}}$. A two-dimensional (2D) tube geometry (Fig 3) was created to mimic realistic AFD standing wave tube measurement with inbuilt air (STP) as the material occupying the fluid domain in the tube geometry. The geometry consisted of a $20 \mathrm{~mm}$ thick, hard backed porous structure (PS), $40 \mathrm{~mm}$ thick fluid domain zone (FD) and 20 $\mathrm{mm}$ thick perfectly matched layer (PML). The geometry was chosen so that simulations could, as best possible, be compared with experimental measurements made in the literature $[8,9]$.
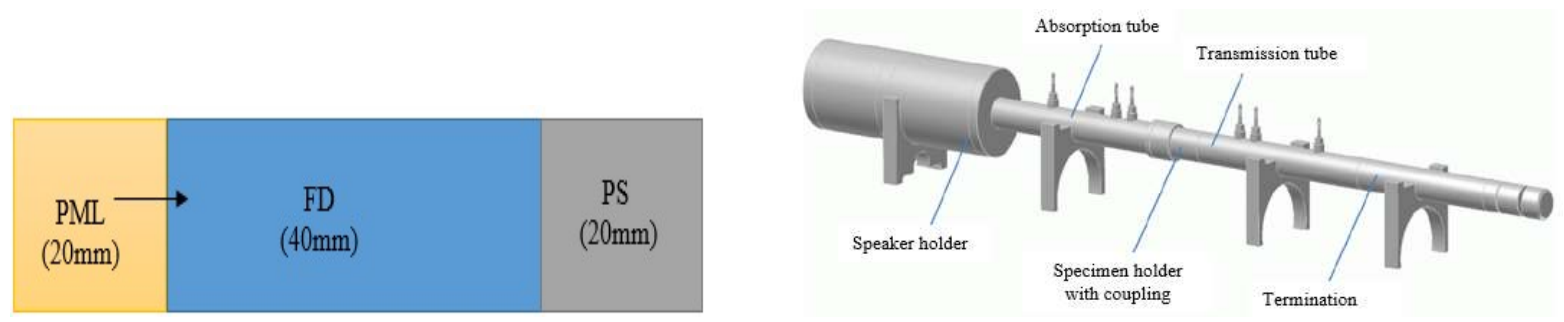

Fig 3. Left, two-dimensional geometrical representation of a hard-backed porous structure in an impedance tube. Right is a schematic of a 4-microphone, AFD 1200-AcoustiTube ${ }^{\circledR}$-measuring setup, with sample holding section (adapted from [15]).

A triangular mesh with $21 \mu \mathrm{m}$ minimum elemental size, maximum elemental size half the minimum wavelength, a maximum element growth rate of 1.3 , curvature factor of 0.3 and 1.0 resolution in the narrow region, were applied to the FD and PS domains while the PML domain was confined to mapped mesh. A Helmholtz or linear acoustic pressure frequency domain was solved on the PML and FD domains. The structural domain (PS) features an interchange 
between equivalent fluid models to account for their real specific surface acoustic impedance and normal incidence absorption coefficient, $A c$, using the characteristic impedance of the materials and the specific surface acoustic impedance of air. A background pressure field was applied on the FD domain, perfectly matched layer on the PML domain, whilst a periodic floquet boundary condition was applied on the side faces. The inlet of PML and outlet of the PS were set as a sound hard boundary wall. The computational time needed to resolve a solution is dependent on the number and range of frequencies considered, typically, 65 frequencies (from 100 to $6500 \mathrm{~Hz}$ ) were resolved in roughly 2 minutes for the above specifications.

In addition to absorption coefficient data across the range of frequencies investigated, several other key parameters were determined in order to enable quantitative assessment and comparison of the acoustic performance of the porous materials. These were: the arithmetic mean of the sound absorption coefficient at the quarter wavelength layer resonance frequencies $(250,500,1000$ and $2000 \mathrm{~Hz})$, also known as the Noise Reduction Coefficient (NRC); the average of the absorption spectra for the twelve one-third octave $(200-2500 \mathrm{~Hz})$ band, also known the Sound Absorption Average $(S A A)$ and the quarter wavelength hard backed layer resonance peak in absorption $(A p)[16]$.

Preliminary tests on the influence of FD (air domain) length showed little or no difference in the simulation results. Similarly, there was very little difference (a 99.2\% correlation) between absorption coefficients measured in $2 \mathrm{D}$ and $3 \mathrm{D}$ simulations, across the whole range of frequencies, justifying the choice of adopting a simpler 2D approach.

\section{Structural characterization}

Models of the acoustical properties of porous media, such as those by Attenborough $[17,18]$, Delany-Bazley-Miki (DBM) [19], and Wilson [22], require the input of the key parameters that define the acoustic behaviour for the different materials. These comprise, the airflow resistivity $(\sigma)$, open porosity $(\varepsilon)$ and high-frequency limit of dynamic tortuosity $(\tau)$. Rigorous definitions for these terms can be found in [21, 23]. Capturing the effects imposed by viscous losses 
(openings) and thermal conduction (pore sizes) in rigid-porous samples saturated with a Newtonian fluid was reported in [24] to give an accurate description of the acoustic behaviour of a motionless skeleton having arbitrary pore shape. A fourth parameter, viscous characteristic length $(\wedge)$, was further introduced to account for the visco-inertial dissipation mechanism of the porous sample. The thermal dissipation mechanism was accounted for, using an additional parameter, the thermal characteristic length $(\pi)$. The inclusion of the viscous [21] and thermal characteristic [20] lengths in the equivalent fluid models for rigid-porous materials gave rise to to the Johnson-Champoux-Allard (JCA) model.

For the porous structures chosen to aid in the development of the simulation methodology, values for these parameters exist. This is not the case for porous metals with bottleneck structures. In the absence of directly defined values in the literature, the airflow resistivity was calculated from the ratio of the fluid dynamic viscosity (for air, $\mu \sim 1.8205 \times 10^{-5} \mathrm{~Pa}$.s) to the viscous permeability $\left(k_{0}\right)$ of the porous material, in the Darcy regime. In the absence of models for the high-frequency limit of dynamic tortuosity that directly embody the geometrical features for bottleneck structures, a best approximation was sought using the well-established approach in $[25,26]$. This model uses a rectangular representation of the solid micro geometry as a representative volume element (RVE) of the internal structure of a porous metal, expressing the tortuosity $(\tau)$ in [25] as a function of the pore volume fraction (porosity, $\varepsilon$ ) using Eqn 1. By calculating the root of this equation, the tortuosity model was explicitly written in $[25,26]$ using Eqn 2. The viscous characteristic length $(\wedge)$ and thermal characteristic length $(\pi)$ were estimated to be half the pore and window sizes respectively $[21,27]$.

$$
\begin{aligned}
& \frac{\tau}{\varepsilon}=\frac{4}{(3-\tau)^{2}} \\
& \text { Eqn } 1 \\
& \tau=2+2 \cos \left[\frac{4 \pi}{3}+\frac{1}{3} \cos ^{-1}(2 \varepsilon-1)\right]
\end{aligned}
$$

The Johnson-Champoux-Allard-Prides-Lafarge (JCAPL) semi-phenomenological model [28, 29] is applicable to arbitrary pore geometries and can account for constrictions between these pores, but requires the determination of 3 additional parameters that are much more complex to measure. A more practical way to estimate them is to use dimensionless shape factors [23]. 
For structures with identical circular pores (as in the ones presented herein and in [9]), these shape factors are equal or very close to unity [28] and the JCAPL model reduces to the JCA model.

Pore-structure characterization is possible using geometrical representation of the porous structure, derived through either simple packing models $[30,31]$ or directly from porous samples using X-ray tomography [15, 32], coupled with modelling of transport through these structures. In this way, all the parameters required for the JCAPL model [31] can be estimated. The process is highly complex, and not without observed inaccuracies, but it does affirm [31] the close correlation between the pore and window geometry in bottleneck structures and estimates for the thermal and viscous characteristic lengths in [21,27].

\section{Validation of the simulation approach}

The appropriateness and accuracy of models, and the simulation environment, were tested by comparing simulation data with experimental data available in the literature. Measured and simulated data for normal incidence absorption spectra for $25 \mathrm{~mm}$ hard backed glass wool fiber [11], $25.5 \mathrm{~mm}$ hard backed porous melamine foam [11] and $23.31 \mathrm{~mm}$ hard backed, sintered fiber metal [8] structures are presented in Fig 4. Structural data for the determination of the characteristic sound absorption spectra for these structures, along with the calculated values for their quarter wavelength layer resonance peak in absorption $(A p)$, noise reduction coefficient $(N R C)$ and sound absorption average $(S A A)$ are presented in Table 1.

Table 1. Pore structure-related (literature) and acoustic (simulated) data for porous structures.

\begin{tabular}{l|l|ccccc|ccc}
\hline Literature & Material type & $\varepsilon$ & $\begin{array}{c}\Lambda \\
(\mu \mathrm{m})\end{array}$ & $\begin{array}{c}\pi \\
(\mu \mathrm{m})\end{array}$ & $\begin{array}{c}\mathrm{k}_{0} / 10^{-9} \\
\left(\mathrm{~m}^{2}\right)\end{array}$ & $\tau$ & Ap & NRC & $S A A$ \\
\hline $\begin{array}{l}\text { Kino and } \\
\begin{array}{l}\text { Ueno, } 2008 \\
{[11]}\end{array}\end{array}$ & $\begin{array}{l}\text { Glass wool } \\
\text { fiber }\end{array}$ & 0.987 & 132 & 237 & 1.084 & 1.009 & 0.965 & 0.369 & 0.347 \\
\hline $\begin{array}{l}\text { Bo and } \\
\text { Tianning, }\end{array}$ & $\begin{array}{l}\text { Porous } \\
\text { sintered fiber } \\
\text { metal }\end{array}$ & 0.909 & 113 & 194 & 0.959 & 1.513 & 0.980 & 0.445 & 0.598 \\
\hline $\begin{array}{l}\text { Kino and } \\
\text { Ueno, } 2008\end{array}$ & $\begin{array}{l}\text { Melamine } \\
\text { foam }\end{array}$ & 0.993 & 199 & 445 & 1.390 & 1.005 & 0.930 & 0.356 & 0.332 \\
\hline
\end{tabular}


The predicted characteristic sound absorption spectra fit well to the experimental data observed for both the glass wool fiber (Fig 4a) and melamine foam (Fig 4b) structures, for both models, with an overall better fit to the Delany-Bazley-Miki (DBM) model. The characteristic absorption spectra for these highly porous materials are reported to depend mainly upon the pore morphology and porosity [7, 11]. The DBM empirical model was specifically developed to model the acoustic behaviour for these relatively simple structures and the good fit, as observed in [11], is expected. The structural morphology of melamine foam is similar to that of commercially available porous metallic structures made by replication of open cell foams, for example Recemat ${ }^{\mathrm{TM}}$, Porvair ${ }^{\mathrm{TM}}$ and Alantum ${ }^{\mathrm{TM}}$ foams [15]. It is not surprising, therefore, that modelling the sound absorption behaviour for these foams is also quite accurate using the Delany-Bazley-Miki (DBM) model [7]. The JCA model gives a better fit to the experimental data recorded for porous sintered fiber metal [8]; the DBM model was not able to accurately predict the behaviour characterized by a dip in the absorption curve for frequencies beyond $3000 \mathrm{~Hz}$. This observation highlights that, as the complexity of the porous structure increases, from transversely isotropic structures, to more complicated structures with increased "strut" thickness, reduced porosity and larger surface area, a greater number of structural terms are required to accurately describe their acoustic behaviour.

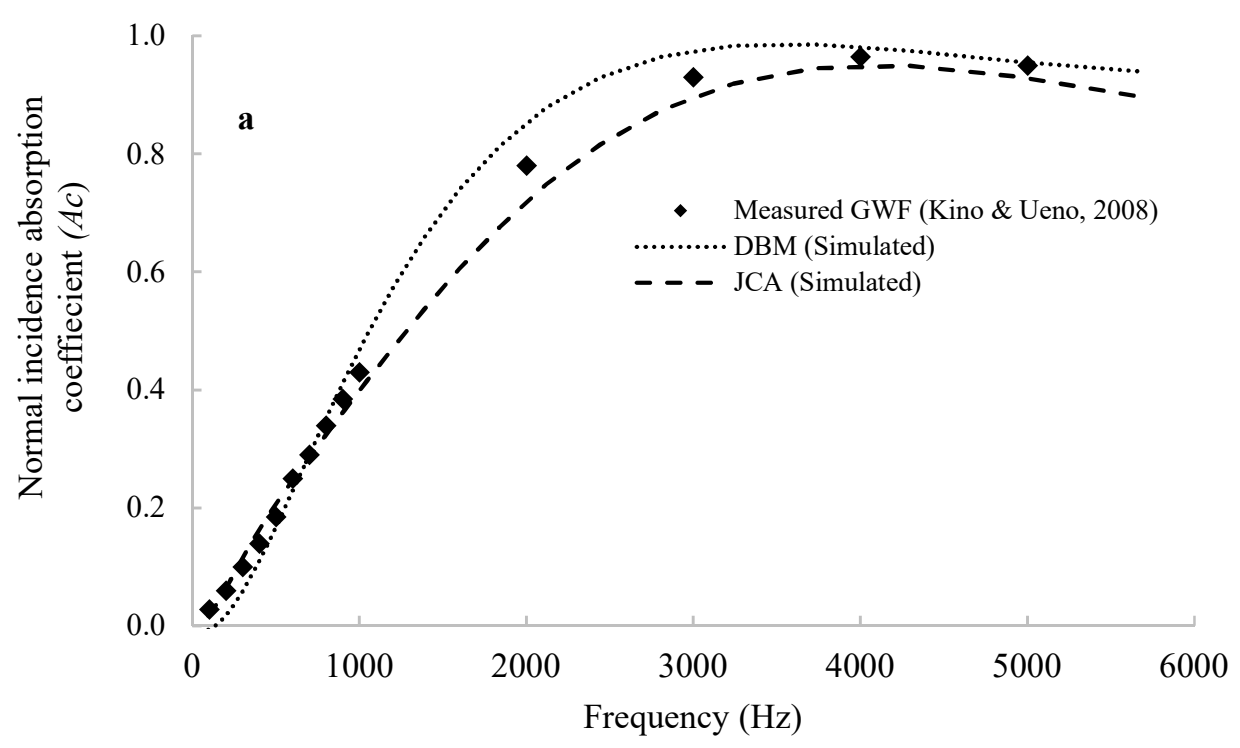



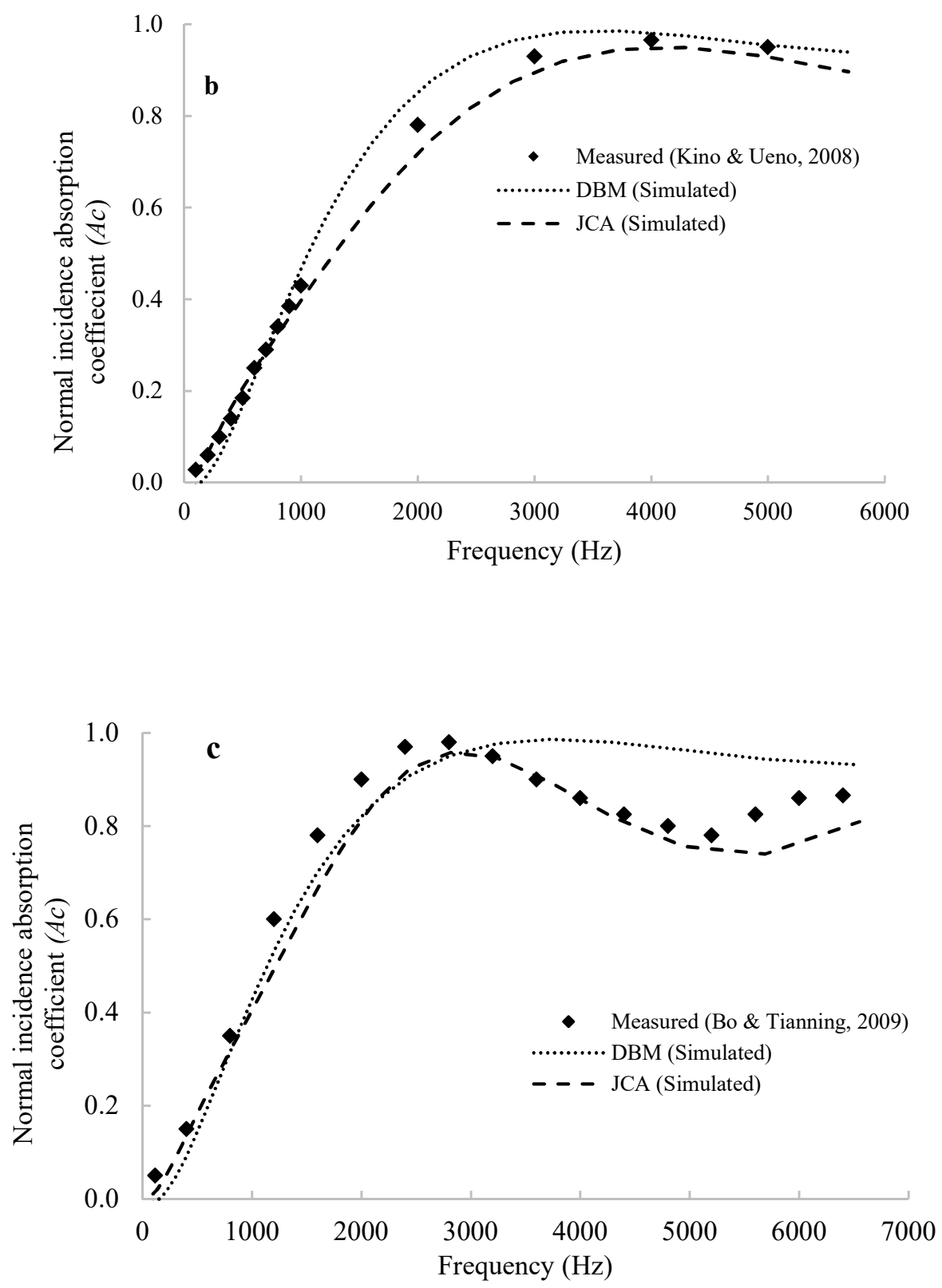

Fig 4. Plots of measured $[8,11]$ and simulated normal incidence absorption spectra for (a) hard backed glass wool fibre (b) hard backed melamine foam (c) hard backed porous sintered fibre metal.

\section{Simulation of sound absorption in bottleneck type porous metal structures}

Fig 5 plots simulation predictions against experimental data for sound absorption in a bottleneck structure [9], data that were also plotted in Fig 1. Input data for these predictions were taken from Table 2, which identifies the assumptions made to enable values to be obtained for all of the parameters required. 
Based on previous observations, it is not surprising that the DBM model does not fit well to the experimental data. The Attenborough model also fails to describe the measured characteristic absorption over almost all the frequency range. A likely cause of this disagreement is that the model $[17,18]$ is a modification of the Zwikker-Kosten [33] equivalent fluid model developed for sand and soil with much lower pore volume fractions.

Table 2. Pore structure-related parameters for porous Al with bottleneck structure in [9].

\begin{tabular}{l|lccc}
\hline Property & Determined by & Value & Units & Model \\
\hline $\begin{array}{l}\text { Pore volume fraction } \\
(\varepsilon)\end{array}$ & Measured in [9] & 0.6 & - & $\begin{array}{l}\text { Attenborough, JCA, } \\
\text { Wilson }\end{array}$ \\
\hline Pore diameter $(d p)$ & Measured in [9] & 1.60 & $\mathrm{~mm}$ & \\
\hline Window diameter $\left(d_{w}\right)$ & Measured in [9] & 0.31 & $\mathrm{~mm}$ & \\
\hline Permeability $\left(k_{0}\right)$ & Measured in [9] & $0.81 \times 10^{-09}$ & $\mathrm{~m}^{2}$ & DBM, Attenborough, \\
& & & JCA, Wilson \\
\hline $\begin{array}{l}\text { High-frequency limit of } \\
\text { dynamic tortuosity }(\tau)\end{array}$ & Equation 1 & 1.867 & & Attenborough, JCA, \\
\hline Thermal length $(\pi)$ & Half the pore diameter & 800 & $\mu \mathrm{m}$ & JCA \\
\hline Viscous length $(\wedge)$ & Half the window diameter & 155 & $\mu \mathrm{m}$ & JCA \\
\hline
\end{tabular}

The JCA model is also a poor fit to the experimental data for the bottleneck structures. This model was developed for porous materials with arbitrary pore shapes [21, 34] and fits the experimental data well for irregular pore networks with high porosity, as evidenced by the good fit to the sintered metal fibre structure data in Fig 4. Fig 5 shows that the Wilson relaxation model [22] is the best and a very close fit to the measured absorption curve. Wilson's relaxation model is functionally quite similar to the JCA [21] model but has at its basis the concept of relaxation times for perturbations to the velocity and thermal fields. The methodology is also distinguished from [21] and similar models, by its approach to accurately describe the behaviour for the boundary layer at the scale of the pore size, where there is a transition in the relaxation behaviour [22]. The resulting relaxation model is barely distinguishable from the exact solution for circular pores, the geometry that most closely represents porosity in bottleneck structures. 


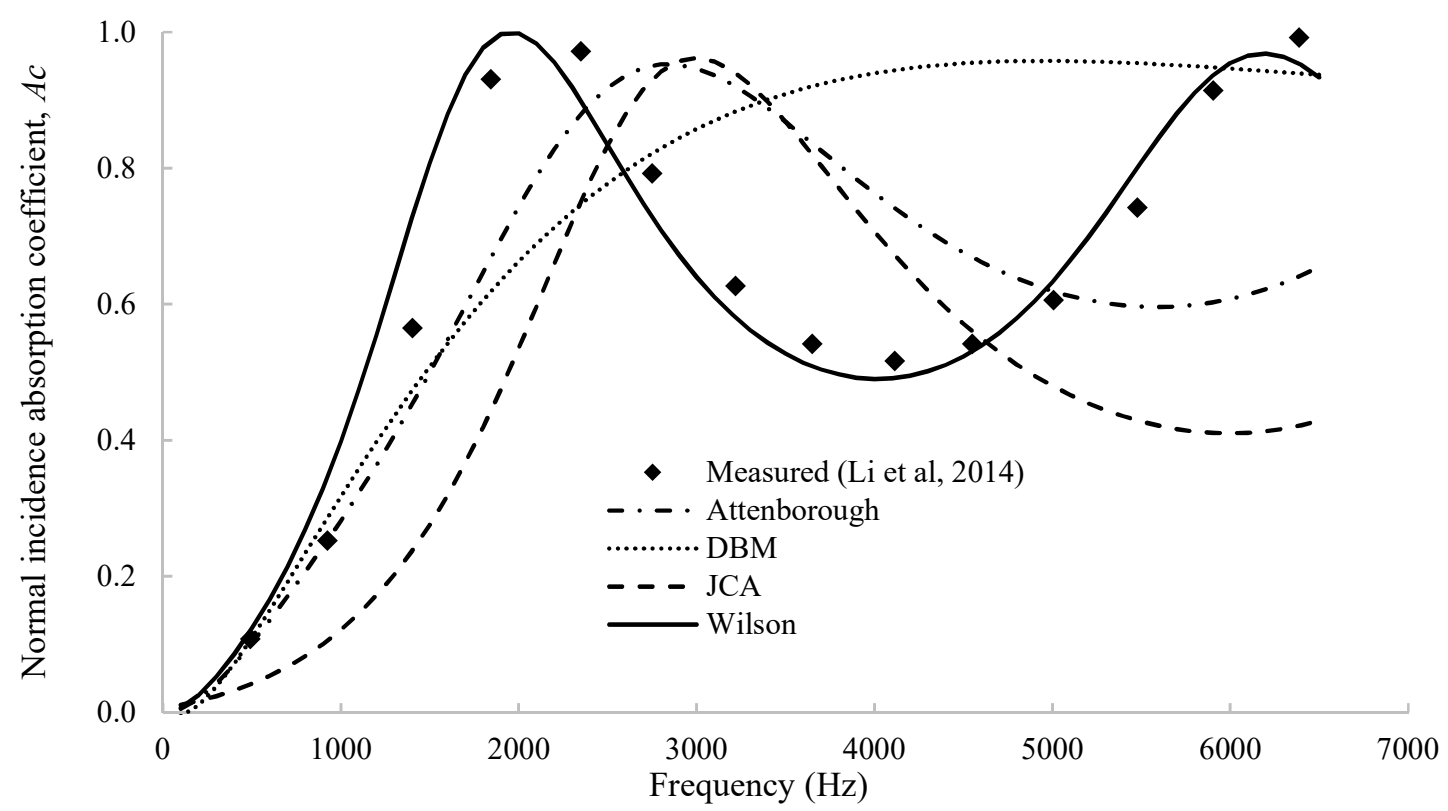

Fig 5. Plots of measured (Li et al., 2014 [9]) and modelled normal incidence absorption spectra ( $A c$ ) for hardbacked $20 \mathrm{~mm}$ thick porous aluminium with a bottleneck structure.

Zielinski [31], using a geometrical representation of the pore structure and transport modelling to determine modelling parameters, used the JCAPL model [28, 29] to estimate, with reasonable accuracy, the sound absorption behaviour for a porous ceramic (pore volume fraction of 0.88 ) with a bottleneck type structure. Fig 6 presents simulations, using parameters determined in [31], to model experimental data in [31], comparing both the JCAPL and Wilson models. It is interesting to note, that although [31] highlights the appropriateness of the JCAPL model, which, owing to the modelling approach taken did not approximate the shape factors to unity and does not reduce to the JCA model predictions, the fit is at least as good for the much simpler Wilson model. The drop-in absorption at higher frequencies is, in fact, predicted more accurately using the Wilson model approach.

Further examples of accurate prediction by the Wilson model, of sound absorption performance for "bottleneck-type" structures, reported in [9, 34], where average deviations between predicted and measured values for $N R C$ and $A p$ were typically $<5 \%[35,36]$, lend strong support to its applicability to predict sound absorption in porous metals with bottleneck structures. 


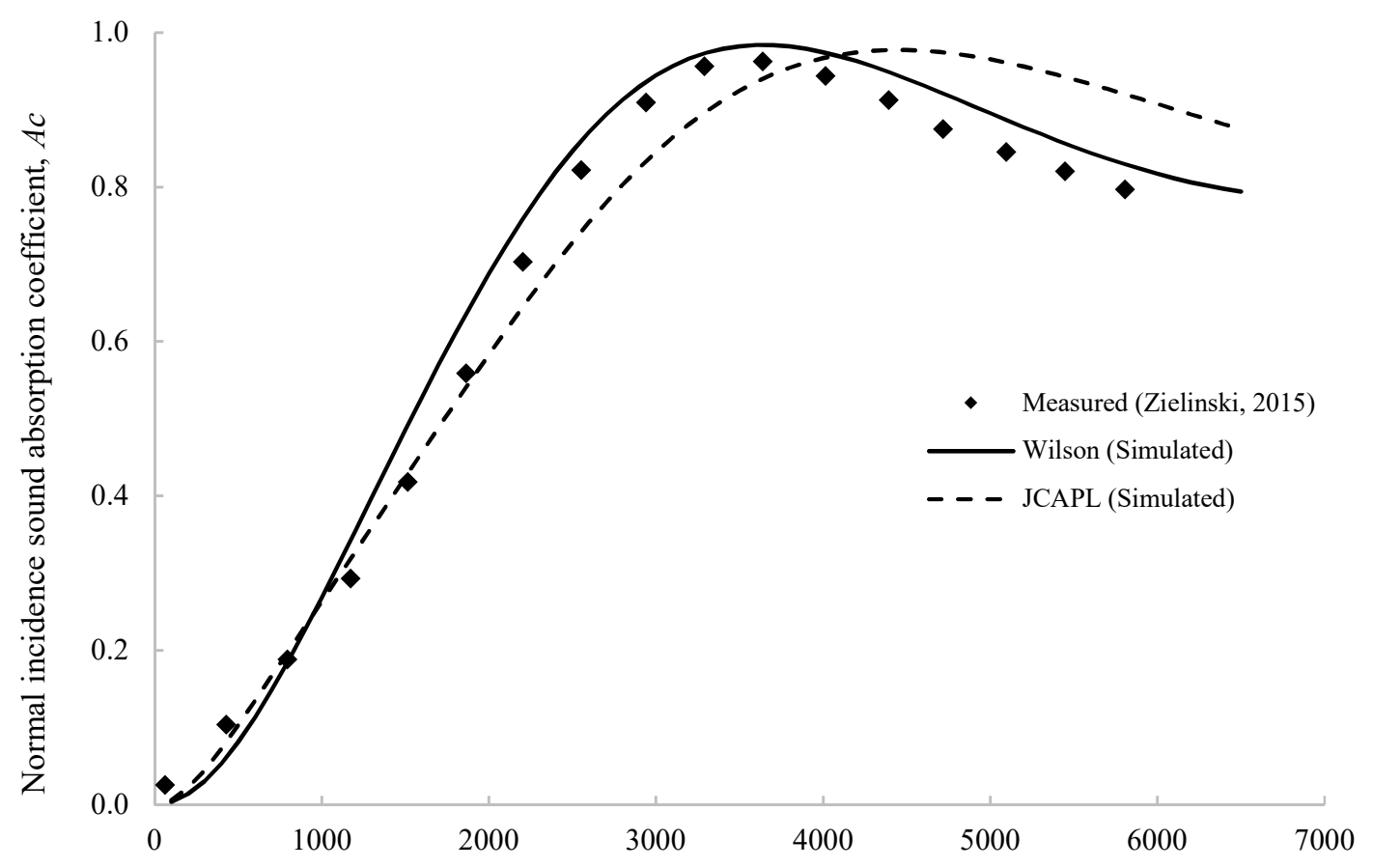

Frequency, $\mathrm{Hz}$

Fig 6. Plots of measured [31] and simulated normal incidence absorption coefficient for "bottleneck-type" ceramic foam structures characterised by pore volume fraction of 0.88 .

\section{Use of the Wilson model for design for sound absorption}

The Wilson model was used to evaluate the potential to improve the sound absorption behaviour of porous metals with bottleneck type structures, assessing the influence of key structural parameters. For the Wilson model, the porosity, tortuosity and permeability must be defined and are controlled by the pore size, porosity and window size. Eqn 2 predicts the effect of porosity on tortuosity. A simple analytical model, derived from the "packing" of monosized spherical pores, connected by windows, is presented in Eqn 3, and has been shown to accurately predict Darcian permeability in "bottleneck" porous metal structures [37-39]. This "bottleneck" permeability model (Eqn 3) considers the contribution of the coordination number for packing $\left(N_{c}\right)$, porosity $(\varepsilon)$, pore $\left(d_{p}\right)$ and window $\left(d_{w}\right)$ diameters for various particle contact cases, such as packing enhanced through compaction [37] and loose packing [38]. An expression for $N_{c}$ in terms of the pore-structure related parameters of virtually-created "bottleneck" structures was reported in [36] and derived by fitting particle packing data in [10, 30] to the macroscopic structural parameters for the porous medium. 


$$
k_{0}=\frac{1.03 N_{c} \varepsilon d_{w}^{3}}{d_{p}} \quad \text { where } N_{c}=17 \varepsilon\left[\frac{d_{w}}{d_{p}}\right]^{0.27} \quad \text { Eqn } 3
$$

In this brief analysis, the porosity and permeability were varied within limits that might be realistically achieved in bottleneck type porous metals [10,32]. Table 3 presents the structural parameters varied and the simulated sound absorption performance, comparing it with the benchmark outlined in Table 1 [9]. Figure 7 shows the normal incidence absorption spectra for the experimental data in [9], along with those for some of the simulated structures. The top figure shows that the effect of increasing the porosity at constant permeability, and to decrease the high-frequency limit of dynamic tortuosity [20], is to effectively decrease the sample thickness and shift the quarter wavelength hard-backed layer resonance to higher frequencies. A benefit of increasing porosity is to minimise the size of the "dip" in $A c$ at higher frequencies, pushing the minimum to a higher frequency.

Utilising the maximum porosity attainable in these types of structures, Fig 7 also plots the effect of varying the permeability. With decreasing permeability, there is a small reduction in $\mathrm{A} p$, but a progressive flattening of the curve at frequencies above the peak value. An optimum balance, for the limited parameters explored here, is achieved for a permeability of $4.0 \times 10^{-10}$ $\mathrm{m}^{2}$, for which a significant overall improvement in sound absorption performance is predicted compared to the benchmark.

Predictions show the clear importance of airflow resistivity or permeability on the sound absorption behaviour, affecting the two relaxation times central to the model. In the Wilson approach, unlike in the JCA model $[20,21]$, the pore and widows sizes do not directly affect the absorption behaviour, rather they "contribute" to the permeability. The "optimum" permeability could, in principle, be achieved through multiple combinations of pore and window geometries. This intuitively, seems unlikely and additional acoustic data are needed to verify this more completely.

Simulations clearly show the potential for enhancing the sound absorption performance. Optimum response is indicated for the maximum porosity, which will be at the expense of 
mechanical performance, such as strength and stiffness. An optimum permeability also exists, lower isn't always better. Eqn 3 highlights the much stronger dependence of the permeability on the window size than the pore size and, as remarked in [24, 40, 41], it is a key parameter governing the sound absorption coefficient in these porous structures. Further detailed simulations using the Wilson model could be applied, defining the porosity to enable mechanical function to be achieved and using the pore and window sizes to optimise the acoustic response via the permeability.

Table 3. Pore structure and acoustic parameters for bottleneck porous structures, simulated using the Wilson model.

\begin{tabular}{ccccccc}
\hline Samples & $\varepsilon$ & $\tau$ & $k_{0} / \mathrm{m}^{2}$ & $A p$ & NRC & $S A A$ \\
& & & & & & \\
\hline V0 [9] & 0.6 & 1.867 & $8.1 \times 10^{-10}$ & 0.998 & 0.390 & 0.593 \\
VA & 0.7 & 1.743 & $8.1 \times 10^{-10}$ & 1.000 & 0.378 & 0.579 \\
VB & 0.8 & 1.575 & $8.1 \times 10^{-10}$ & 0.998 & 0.377 & 0.581 \\
\hline VC & 0.5 & 2.001 & $8.1 \times 10^{-10}$ & 0.989 & 0.336 & 0.511 \\
VD & 0.8 & 1.575 & $10.0 \times 10^{-10}$ & 0.987 & 0.361 & 0.556 \\
VE & 0.8 & 1.575 & $6.0 \times 10^{-10}$ & 0.997 & 0.397 & 0.609 \\
VF & 0.8 & 1.575 & $4.0 \times 10^{-10}$ & 0.969 & 0.418 & 0.631 \\
VG & 0.8 & 1.575 & $2.0 \times 10^{-10}$ & 0.841 & 0.429 & 0.611 \\
\hline
\end{tabular}

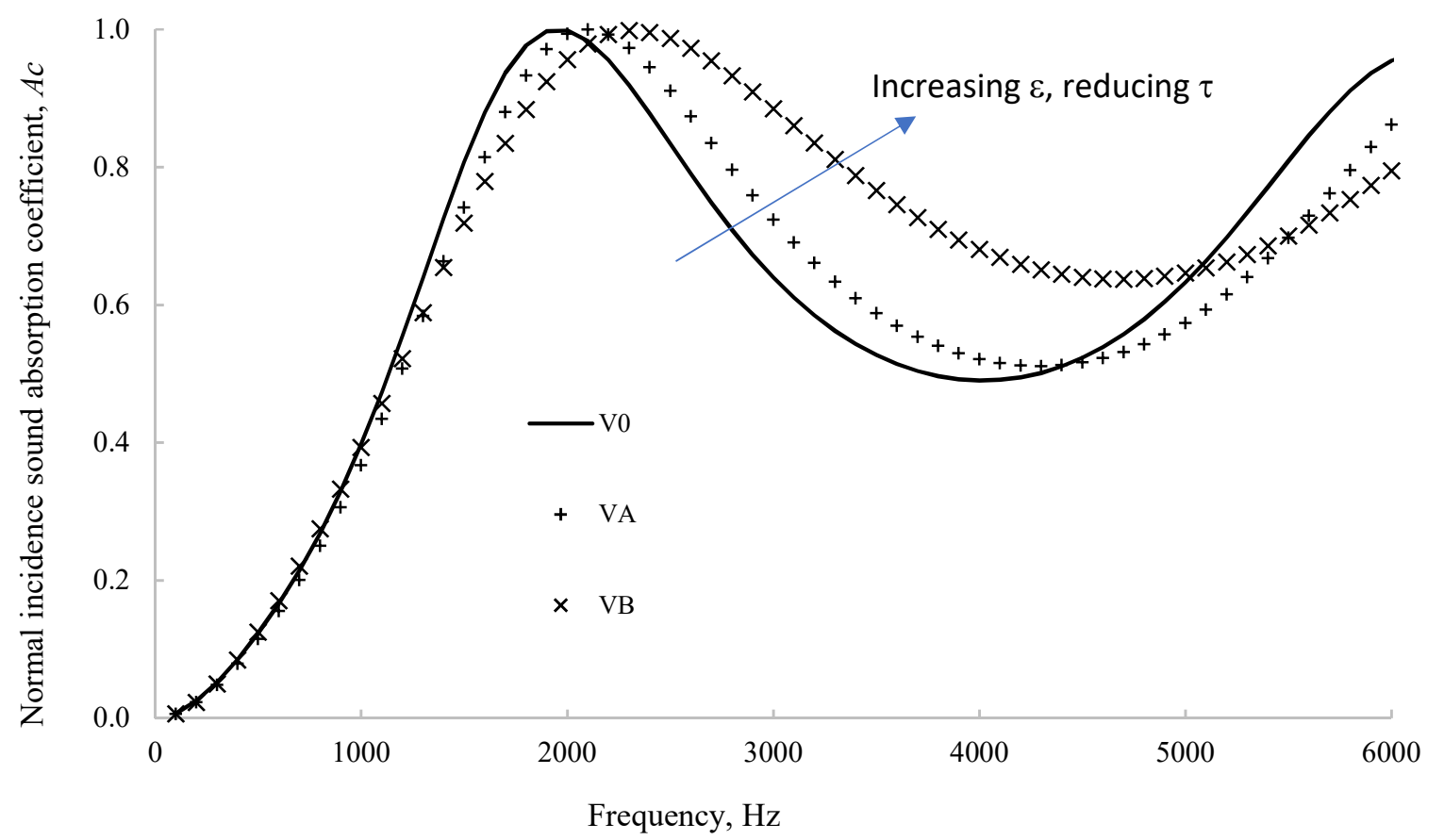




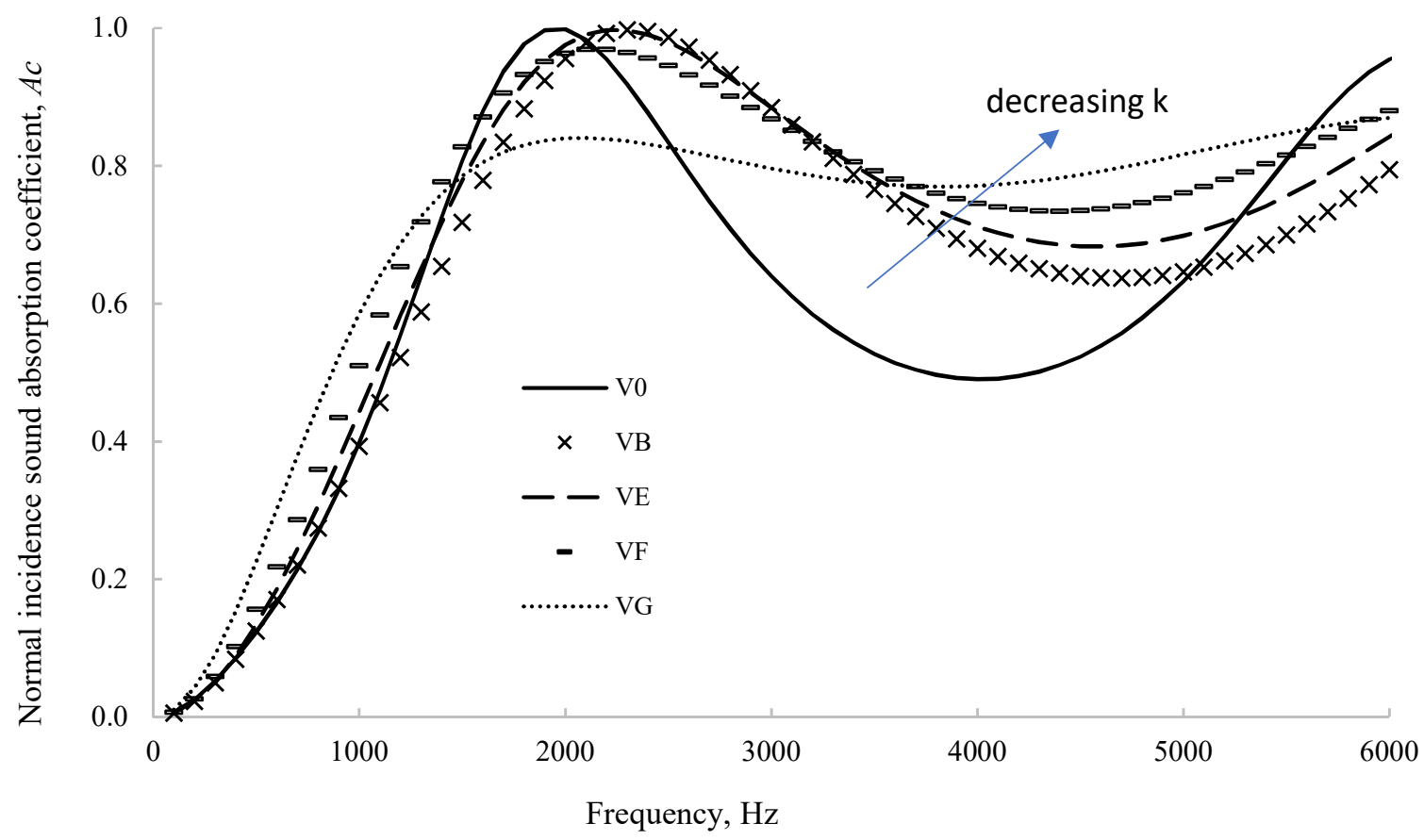

Fig 7: Plots of simulated normal incidence absorption spectra (Ac) against frequency, comparing experimental data [9] with predicted performance for a variety of bottleneck type structures. Top, the effect of varying porosity (and tortuosity) at constant permeability, bottom, the effect of varying permeability at constant porosity (and tortuosity).

\section{Conclusions}

Wilson's relaxation model was found to be an excellent fit to experimental acoustic absorption data for porous materials with bottleneck type structures and an overall best fit when compared to other models of acoustical properties of porous media.

Model predictions were able to highlight the relative importance of porosity, tortuosity and permeability on the quarter wave resonance peak in absorption and the normal incidence sound absorption spectrum.

Simulations revealed significant improvements in absorption behaviour over "benchmark" structures can be expected by maximising the porosity $(0.8)$ and targeting a permeability in the range of $4.0 \times 10^{-10} \mathrm{~m}^{2}$.

Such a modelling approach can provide a valuable tool in the optimisation of sound absorption performance and structural integrity to meet application-specific requirements, for a genre of 
porous materials that offer a unique combination of acoustic absorption and load bearing capability.

Acknowledgments: OAJ would like to thank the University of Nottingham, Nottingham, United Kingdom, Simpleware - Synopsys Technology, United Kingdom and Bowers \& Wilkins Group, West Sussex, United Kingdom, for the provision of funds, licenses and technical support needed for the successful completion of this work.

\section{References}

1 Jorges, P. A. and Malcom, J. C. (2010), Recent-Trends in Porous Sound-Absorbing Materials, Sound and Vibration, Vol 44, Pp 12-17.

2 Deng, X. Z., Liu, H. S. and Huang, X. Y. (2008), Numerical Simulation of Effect of $\mathrm{CO}_{2}$ Content on Cell Nucleation in Microcellular Plastics Extrusion Processing for Supercritical CO2/PS, Plastics Sci. Tech. 36(8), Pp 30-32.

3 Xiaolin, W., Feng, P. and Baojun, C. (2009), Sound Absorption of Porous Metals at High Sound Pressure Levels, Journal of Acoustic Society of America, 126 (2).

4 F. García-Moreno, Commercial applications of metal foams: their properties and production, Materials 9 (2) (2016) 85.

5 J Banhart, Manufacture, characterisation and application of cellular metals and metal foams, Progress in Materials Science, Volume 46, Issue 6, 2001, p 559-632.

6 A Kennedy. (2012), Porous Metals and Metal Foams Made from Powders, Powder Metallurgy, Katsuyoshi Kondoh, Intech Open, DOI: 10.5772/33060.

7 Hinze, B. and Rosler, J. (2014), Measuring \& Simulating Acoustic Absorption of Open-Celled Metals. Advanced Engineering Materials, 16. No 3.

8 Bo, Z. and Tianning, C. (2009), Calculation of Sound Characteristics of Porous Sintered Fiber Metal. Applied Acoustics 70, Pp 337-346.

9 Li, Y., Zhendong, L. and Han, F. (2014). Airflow Resistance and Sound Absorption Behaviour of Opencelled Aluminium Foams with Spherical Cells. Procedia Materials Science Vol 4, Pp 187-190.

10 Langston, P. and Kennedy, A. R. (2014). Discrete Element Modelling of the Packing of Spheres and its Application to the Structure of Porous Metals made by Infiltration of Packed Beds of $\mathrm{NaCl}$ Beads. Powder Technology, 268, Pp 210-218.

11 Kino, N. and Ueno, T. (2008), Comparison between Characteristic Lengths and Fiber Equivalent Diameter in Glass Fiber and Melamine Foam Materials of Similar Flow Resistivity, J. App. Acoustics, Vol 69, P. 325.

12 Han, F., Seiffert, G., Zhao, Y. and Gibbs, B. (2003), Acoustic absorption Behaviour of an Open-celled Aluminium Foam. J. Phys. D, 36, Pp $294-302$.

13 Li, Y., Wang, X., Wang, X., Ren, Y., Han, F., and Wen C., (2011), Sound Absorption Characteristics of Aluminium Foam with Spherical Cells, Journal of Applied Physics 110, 113525.

14 Wang, X., and Lu, T. J. (1999), Optimized Acoustic Properties of Cellular Solids, The Journal of the Acoustical Society of America, Vol, 106, Pp 756-763.

15 Otaru, A.J. (2019), Enhancing the Sound Absorption Performance of Porous Metallic Structures using Tomography Images, Applied Acoustics, Vol 143, Pp 183-189.

16 Bell, L. H. (1982), Industrial Noise Control. Marcel Dekker, New York, P 199.

17 Attenborough, K. (1983), Acoustical Characteristics of Rigid Fibrous Absorbents and Granular Materials, $J$. Acoust. Soc. Am., Vol 73, Pp 785-799.

18 Attenborough, K. (1993), Models for the Acoustical Characteristics of Air-Filled Granular Materials, Acta Acoustica, Vol 1, Pp213-226.

19 Miki, Y. (1990), Acoustical Properties of Porous Materials-Modifications of Delany Bazley Model, J. Acoust. Soc., Jpn (E). 11(1), Pp 19-24.

20 Champoux, Y. and Stinson, M.R. (1992), On Acoustical Models for Sound Propagation in Rigid Frame Porous Materials and the Influence of Shape Factors, J. Acoust. Soc. Am. Vol 92(2), Pp1120-1131. 
21 Johnson, D. L., Koplik, J. and Dashen, R. (1987), Theory of Dynamic Permeability \& Tortuosity in FluidSaturated Porous Media, J. Fluid Mech., Vol 176, Pp 176-402.

22 Wilson, D. K. (1993), Relaxation-Matched Modelling of Propagation through Porous Media, Including Fractal Pore Structure, J. Acoust. Soc. Am., Vol 94(2), Pp 1136-1145.

23 Dukhan, N. (2013), Metal Foams: Fundamental and Applications. DESTECH Publication, Inc. Technology \& Engineering, USA, Pp 1-310.

24 Chevillotte, F. and Perrot, C. (2017), Effect of the Three-Dimensional Microstructure on the Sound Absorption of Foams: A Parameteric Study, J. Acoust. Soc. Am., Vol 142(2), Pp 1130-1140.

25 Smith, G.J.F. and Du Plessis, J.P. (1999), Modelling of non-Newtonian Purely Viscous Flow through Isotropic High Porosity Synthetic Foams, Chem. Eng. Sci. Vol 54, Pp 645-654.

26 Du Plessis, J. P. and Fourier, J. G. (2002), Pressure Drop Modelling in Cellular Metallic Foams, Chem. Eng. Sci. Vol 57, Pp 2781-2789.

27 Olny, X. and Panneton, R. (2008), Acoustic Determination of the Parameters Governing Thermal Dissipation in Porous Media, J. Acoust. Soc. Am., Vol 123, Pp 814-824.

28 Lafarge, D., Lemarinar, P. and Allard, J. F. (1997), Dynamic Compressibility of Air in Porous Structure at Audible Frequencies, Journal of Acoustic Society of America, Pp 1-12.

29 Prides S. R., Morgan F. D. And Gangi A. F. (1993), Drag Forces of Porous-Medium Acoustics, Phys. Rev. $B$ Vol 47, Pp 4964-4978.

30 Otaru, A. J. and Kennedy, A. R. (2016), The Permeability of Virtual Macroporous Structures Generated by Sphere-Packing Models: Comparison with Analytical Models. Scripta Materialia, Vol 124, Pp 30-33.

31 Zielinski , T.G. (2015), Generation of Random Microstructures and Prediction of Sound Velocity and Absorption for Open Foams with Spherical Pores, J. Acoust. Soc. Am., Vol 137 (4), Pp 1790-1801.

32 Otaru, A.J., Morvan, H. P. and Kennedy, A. R. (2018), Measurement and Simulation of Pressure Drop across Replicated Microcellular Aluminium in the Darcy-Forchheimer Regime, Acta Materialia, Vol 149, Pp 265275.

33 Zwikker, C. and Kosten, C.W. (1949), Sound Absorbing Materials, Elsevier, New York.

34 Lu. T. J. F Chen and He, D. (2000), Sound Absorption of Cellular Metals with Semi-Open Cells, J. Acoust. Soc. Am. 108 (4), Pp 1697-1708.

35 Otaru, A.J., (2018), Fluid Flow and Acoustic Absorption in Porous metallic Structures using Numerical Simulation and Experimentation, PhD Thesis, The University of Nottingham, United Kingdom.

36 Otaru, A.J., Morvan, H.P. and Kennedy, A.R. (2018), Modelling and Optimisation of Sound Absorption in Replicated Microcellular Metals, Scripta Materialia, Vol 150, Pp 152-155

37 Despois, J. F., and Mortensen, A. (2005), Permeability of Open-pore Microcellular Materials, Acta Materialia 53, Pp 1381-1388.

38 Furman, E. L. Finkelstein, A .B. and Cherny, M. L. (2013), The Permeability of Aluminium Foams Produced by Replicated-Casting. Metals. Vol 3, Pp 49-57.

39 Otaru, A.J., Morvan, H.P. \& Kennedy, A.R. (2019), Airflow Measurement across Negatively Infiltration Processed Porous Aluminium Structures, AIChE Journal, https://doi.org/10.1002/aic.16523

40 Chevillotte, F., Perrot, C. and Panneton, R. (2010), Microstructure Based Model for Sound Absorption Predictions of Perforated Closed-cell Metallic Foams, J. Acoust. Soc. Am., Vol 128(4), Pp 1766-1776.

41 Perrot, C., Chevillotte, F. and Panneton, R. (2008), Bottom-up Approach for Microstructure Optimisation of Sound Absorbing Materials, J. Acoust. Soc. Am., Vol 124(2), Pp 940-948. 